\title{
Solar and stellar coronae and winds
}

\author{
Moira Jardine \\ SUPA, School of Physics and Astronomy, University of St Andrews, North Haugh, \\ St Andrews, KY16 9SS, UK \\ email: mmj@st-andrews.ac.uk
}

\begin{abstract}
Solar-like stars influence their environments through their coronal emis- sion and winds. These processes are linked through the physics of the stellar magnetic field, whose strength and geometry has now been explored for a large number of stars through spectropolarimetric observations. We have now detected trends with mass and rotation rate in the distribution of magnetic energies in different geometries and on also different length scales. This has implications both for the dynamo processes that generate the fields and also for the dynamics and evolution of the coronae and winds. Modelling of the surface driving processes on stars of various masses and rotation rates has revealed tantalising clues about the dynamics of stellar coronae and their ejecta. These new observations have also prompted a resurgence in the modelling of stellar winds, which is now uncovering the range of different interplanetary conditions that exoplanets might experience as they evolve.
\end{abstract}

Keywords. stars:magnetic fields, stars:coronae, stars:imaging, stars:spots

\section{Introduction}

In the following I aim to focus mainly on the relationship between stellar coronae and winds, and in particular to explore the ways in which this is governed by the stellar magnetic field geometry. This relationship can be seen very clearly for the Sun when we look at the variation in the coronal X-ray emission and wind speed. At cycle minimum, most of the emission originates close to the equator, where the slow wind originates. The fast wind, in contrast, comes mainly from the polar regions. During the maximum phase of the sunspot cycle, however, a much more complex picture is seen, with fast and slow wind streams interspersed at a range of latitudes. This change in the morphology of both the closed and open field regions is driven by the evolution of the Sun's magnetic field over the course of its cycle. We can analyse these changes by looking at the variation of the dipole and quadrupole modes through the solar cycle. As shown by DeRosa et al. (2012), the strength of the quadrupole contribution varies in phase with the sunspot number, while the strength of the dipole mode is in antiphase (see also Vidotto (2016)).

Clearly, if we are to understand the changes in the Sun's corona and wind through its cycle, we need to model the changes in its magnetic field. There are, of course, many approaches to this - in this paper I will focus on one method that is particularly applicable to stars. The Wang-Sheeley-Arge (WSA) model is an empirical approach, that maps the wind speed directly to the magnetic field geometry (Wang \& Sheeley 1990; Arge \& Pizzo 2000). The magnetic field structure in the corona is extrapolated from surface magnetograms using a potential field source surface method (Altschuler \& Newkirk 1969). An inherent assumption of this model is that the magnetic field is forced open by the coronal pressure at some radius $r_{s}$, known as the source surface. The wind speed is then related directly to the field line expansion factor $f$ as follows:

$$
u_{i}\left[\mathrm{kms}^{-1}\right]=267.5+\frac{410.0}{f_{i}^{2 / 5}}
$$



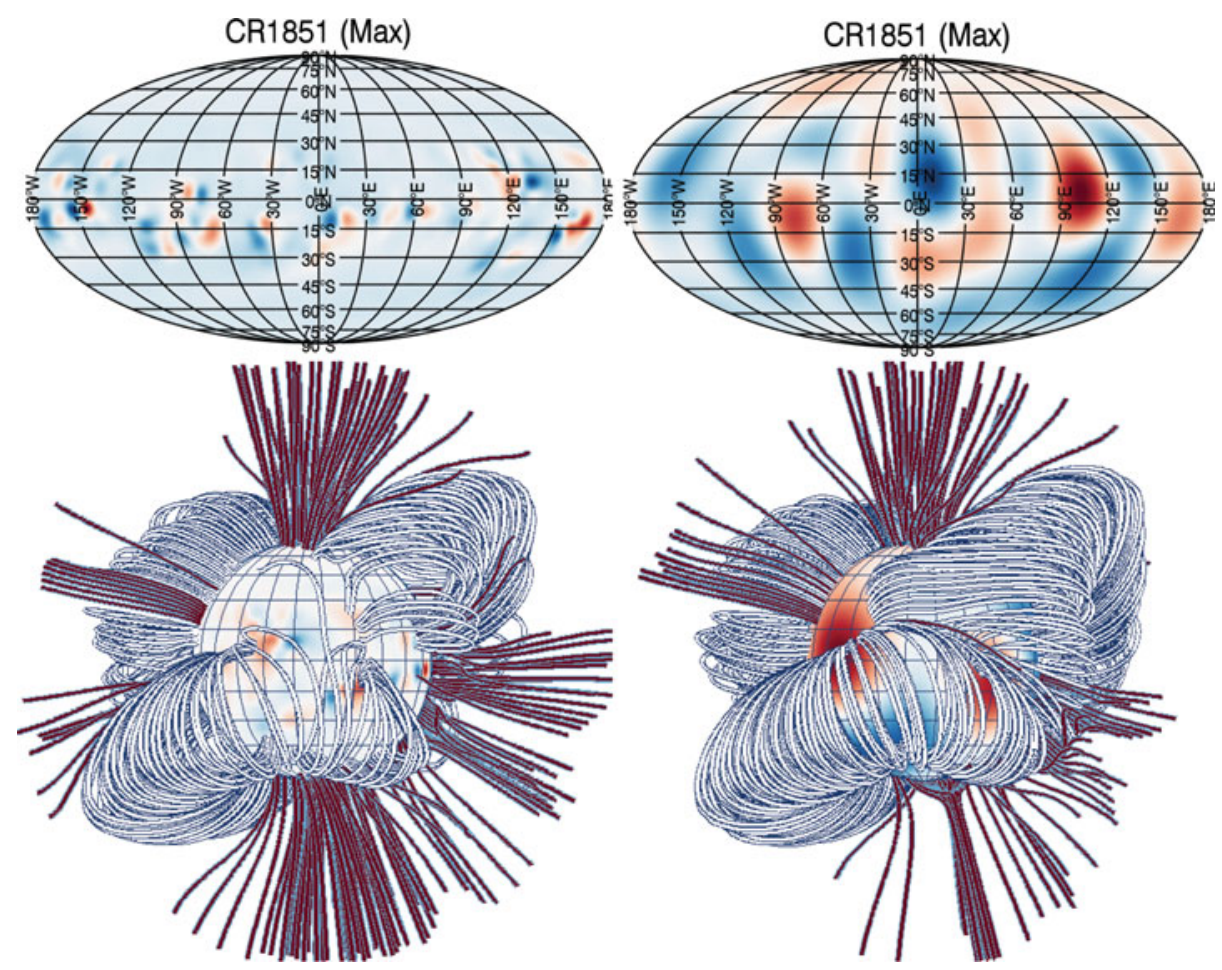

Figure 1. Top: Surface magnetograms for Carrington rotation 1851 (close to cycle maximum). The map is reconstructed for a maximum spherical harmonic degree of (left) $\ell_{\max }=63$ and (right) $\ell_{\max }=5$ corresponding to surface spatial scales of $3^{\circ}$ and $30^{\circ}$ respectively. Colourbars are set to $\pm 200 \mathrm{G}$ (left) and $\pm 30 \mathrm{G}$ (right) . Bottom: The corresponding field extrapolations, with wind-bearing (open) field lines coloured red. The overall structure of the largest fieldlines is very similar.

where the expansion factor $f_{i}$ of any field line is given by:

$$
f_{i}=\frac{r_{\odot}^{2}}{r_{\mathrm{s}}^{2}} \frac{B_{i}\left(r_{\odot}\right)}{B_{i}\left(r_{\mathrm{s}}\right)} .
$$

A large expansion factor therefore leads to a slow wind and conversely a small expansion factor leads to a fast wind. This approach is computationally very efficient and underlies many models of the solar wind. In an attempt to quantify the effect that the observational resolution might have on the output from such a code, Cohen (2015) undertook a detailed comparison of this approach with much more sophisticated MHD models. He showed that for the Sun, an increase in resolution from 2 to 1 in the surface magnetogram leads to only modest changes in the arrival time at Earth of coronal mass ejections. This approach has also been employed in analysing the variation of the solar wind through its cycle that accompanies the changing magnetic field structure (Pinto et al. 2011; Gressl et al. 2014; Pinto et al. 2016).

\section{Application to other stars}

Maps of the surface magnetic fields of other stars have been available now for some time. These show trends in the field strength and geometry with both mass and rotation rate (Donati and Landstreet 2009). It is clear that many stars have surface magnetic 
fields that are very non-solar in nature, and this is likely to have direct implications for the structure and dynamics of their coronae and winds.

\subsection{Resolution}

Extending this method to other stars would therefore be very useful, but faces the problem of the limited resolution of stellar magnetograms. Figure 1 shows a typical solar magnetogram obtained from the US National Solar Observatory, Kitt Peak, and the potential field extrapolation in the corona, compared to what would be found for a typical stellar magnetogram. These magnetograms are reconstructed using the Zeeman Doppler Imaging technique, which recovers only the large scale field. Surface resolutions for stellar magnetograms are typically $20^{\circ}$ to $30^{\circ}$, although they may be as good as $3^{\circ}$. As can be seen from Figure 1 however, the largest scale field lines in both cases have a very similar geometry. This is simply because only the large scale field extends out into the wind region.

The corresponding wind speeds, derived from the WSA model, are shown in Figure 2 (Jardine, Vidotto, and See 2017). It is clear that a surface resolution of better than about $20^{\circ}$ has little influence on the derived wind speed. This suggests that these magnetograms can indeed be used to explore the 3-D structure of stellar winds.

\subsection{Trends with Rossby number}

It has been known for a long time that the behaviour of stellar winds depends on the open flux of magnetic field (Mestel 1968; Mestel \& Spruit 1987; Réville et al. 2015b,a). While this is not directly observable, it can be calculated from the surface magnetogram. See (2017) used the WSA method combined with an expression for the torque (Réville et al. 2015b,a) to determine open fluxes for a large number of stars whose surface magnetic fields had been mapped and to derive a scaling between the angular momentum loss rate and the Rossby number. The low mass stars, i.e. those without a tachocline, do not lie on this relation however. These stars could therefore be expected to spin down only very slowly. It is notable that when the results of 3D MHD simulations are overplotted on this relation, the agreement is very good.

The other parameter that varies very closely with Rossby number is the X-ray luminosity. This suggests a close relationship between the mass loss rate and the X-ray luminosity. Mass loss rates from solar like stars are very difficult to measure, as the winds have a very low density. One indirect method of doing this, developed by Brian Wood, uses the enhanced absorption in the hydrogen wall of stellar astrospheres as a measure of the local mass loss rate (see, for example, Wood et al. (2005)). Low activity stars appear to show a clear relation between wind mass loss rate and X-ray flux such

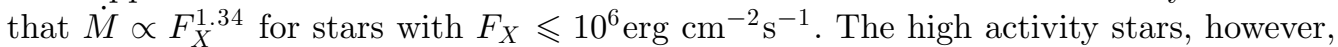
with the largest X-ray flux, seem to lie well below this relation. Vidotto et al. (2016) however showed that there was no clear change in the geometry of the magnetic fields of the stars across this "wind dividing line". Using the mass loss rates derived from the surface magnetograms, See (2017) also found no evidence of a wind dividing line, but recovered a scaling relations $\dot{M} \propto F_{X}^{1.31}$, similar to that found in the observations.

\subsection{Classifying stellar magnetic fields}

The nature of stellar magnetic fields is typically described by their symmetry around the rotation axis, and their geometry. This geometry is expressed by separating the field into its toroidal and poloidal components, as shown in Figure 3. Toroidal fields appear in those stars that have developed a radiative core. See et al. (2015) showed that a plot of the energy in the toroidal field, as a function of the energy in the poloidal field shows 

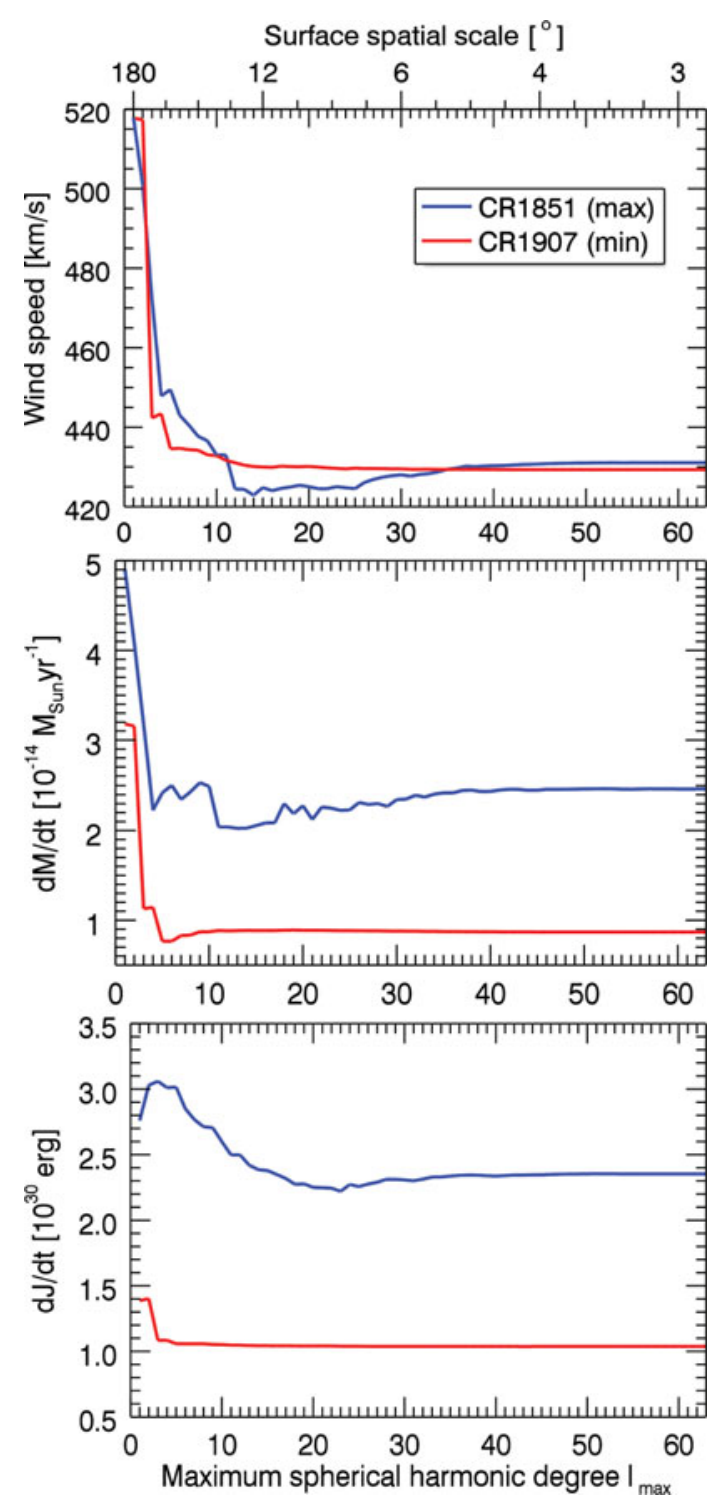

Figure 2. Variation with maximum spherical harmonic degree $\ell_{\max }$ of the average wind speed at the Earth's orbit, the total mass loss rate and the total angular momentum loss rate. Results are shown for two Carrington rotations.

two branches. Stars with radiative cores lie on one branch, while fully-convective stars lie on the other. This suggests that the presence of the tachocline, while not necessary for producing magnetic field, does influence the geometry of a star's magnetic field.

\subsection{Coronal magnetic fields}

The large range in the magnitude of the magnetic energy of these stars suggests that their winds and coronae may be substantially different. In particular the energy release processes may be acting quite differently in more active stars. A comparison between the kinetic energy in solar coronal mass ejections, with the X-ray emission of the associated flare, shows a clear correlation (Drake et al. 2013). Extrapolating this correlation, 


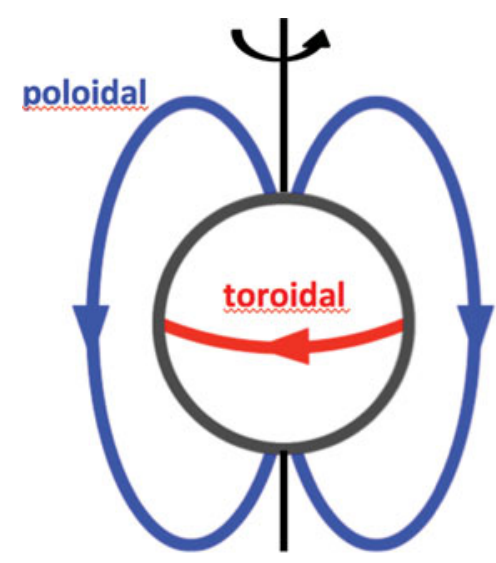

axisymmetric

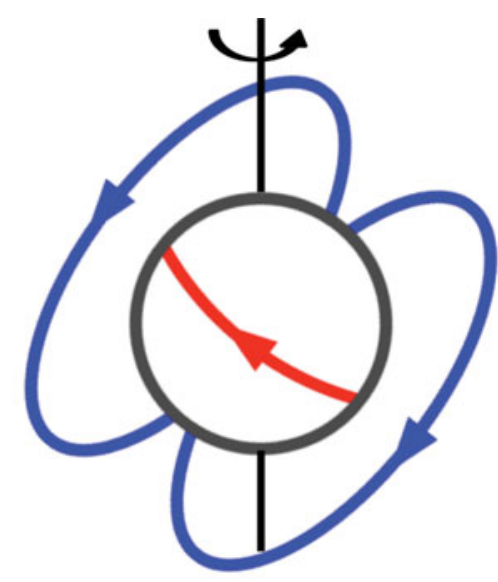

non-axisymmetric

Figure 3. Magnetic fields can be decomposed into poloidal and toroidal components, that may be axisymmetric (left) or non-axisymemetric (right) about the rotation axis.

however, to more active stars, gives an unfeasibly large energy in the coronal mass ejections. So what is happening in the coronae of these very active starts? A pilot study by Gibb, Jardine, and Mackay (2014) suggested that in stars with a large surface shear, the timescales for evolution in the coronae may be surprisingly short. A subsequent largescale study of the roles of flux emergence and differential rotation, showed that while flux emergence governs the the total energy available, it is the surface shear that determines the topology of the magnetic field (Gibb et al. 2016). High shear rates lead to a larger open flux and a higher rate of eruption of flux ropes.

\subsection{Comparing observations and simulations}

Lehmann et al. (2016) have recently analysed the surface magnetic fields of these simulations. They sure that the energies of the simulated magnetic fields lie within the same range as the observed poloidal and toroidal energies. By expressing the field as a sum of spherical harmonics, they were able to truncate the series at different spherical harmonic modes. This allows the field structures of different length scales to be analysed separately. This showed that while the highest order modes (i.e. the small-scale field) have a constant relationship between the toroidal and poloidal energies, the dipole mode is particularly poloidal, while the quadruple mode has the largest relative amount of toroidal field.

\section{Conclusion}

In summary then, it seems that while a tachocline is not necessary for the generation of magnetic field, it may play a role in the geometry of the field that is produced. As a result, it also has a role to play in governing the coronal activity and the efficiency with which the wind can remove angular momentum from the star. The preimpacts both on the evolution of the rotation rate and activity of the star. At any given evolutionary phase for the star, the rate at which flux emerges through the stellar surface governs the total energy available in the corona, but it is the surface shear that controls that geometry of the coronal magnetic field.

Many puzzles remain however. Why does the toroidal field appear so clearly in the quadrupolar component, while the dipole is so poloidal? How is energy distributed on 
the different lengths scales of the magnetic field? These are puzzles that will absorb the community for quite some time.

\section{References}

Altschuler, M. D. \& Newkirk, G. 1969, Solar Phys., 9, 131

Arge, C. N. \& Pizzo, V. J. 2000, JGR, 105, 10465

Cohen, O. 2015, Solar Phys., 290, 2245

DeRosa, M. L., Brun, A. S., \& Hoeksema, J. T. 2012, ApJ, 757, 96

\&onati, J.-F. \& Landstreet, J. D. 2009, Annual Review of Astronomy and Astrophysics 47, 333.

Drake, J. J., Cohen, O., Yashiro, S., \& Gopalswamy, N. 2013, Astrophys. J. 764, 170.

Gibb, G. P. S.., Jardine, M. M., \& Mackay, D. H. 2014, Monthly Notices of the Royal Astronomical Society 443, 3251.

Gibb, G. P. S.., Mackay, D. H., Jardine, M. M., \& Yeates, A. R. 2016, Monthly Notices of the Royal Astronomical Society 456, 3624.

Gressl, C., Veronig, A. M., Temmer, M., et al. 2014, Solar Phys., 289, 1783

Jardine, M., Vidotto, A. A., \& See, V. 2017, Monthly Notices of the Royal Astronomical Society 465, L25.

Lehmann, L. T., Jardine, M. M., Vidotto, A. A., Mackay, D. H., See, V., Donati, J.-F., Folsom, C. P., Jeffers, S. V., Marsden, S. C., Morin, J., \& Petit, P. 2016, ArXiv e-prints, arXiv: 1610.08314 .

Mestel, L. 1968, MNRAS, 138, 359

Mestel, L. \& Spruit, H. C. 1987, MNRAS, 226, 57

Pinto, R. F., Brun, A. S., Jouve, L., \& Grappin, R. 2011, apj, 737, 72

Pinto, R. F., Brun, A. S., \& Rouillard, A. P. 2016, ArXiv e-prints

Réville, V., Brun, A. S., Matt, S. P., Strugarek, A., \& Pinto, R. F. 2015a, ApJ, 798, 116

Réville, V., Brun, A. S., Strugarek, A., et al. 2015b, ApJ, 814, 99

See, V., Jardine, M., Vidotto, A. A., Donati, J.-F., Folsom, C. P., Boro Saikia, S., Bouvier, J., Fares, R., Gregory, S. G., Hussain, G., Jeffers, S. V., Marsden, S. C., Morin, J., Moutou, C., do Nascimento, J. D., Petit, P., Rosén, L., \& Waite, I. A. 2015, Monthly Notices of the Royal Astronomical Society 453, 4301.

See, V. 2017, MNRAS, in press

Vidotto, A. A., Donati, J.-F., Jardine, M., See, V., Petit, P., Boisse, I., Boro Saikia, S., Hébrard, E., Jeffers, S. V., Marsden, S. C., \& Morin, J. 2016, Monthly Notices of the Royal Astronomical Society 455, L52.

Vidotto, A. A. 2016, MNRAS, 459, 1533

Wang, Y.-M. \& Sheeley, Jr., N. R. 1990, ApJ, 355, 726

Wood, B. E., Müller, H.-R., Zank, G. P., Linsky, J. L., \& Redfield, S. 2005, Astrophys. J. 628, L143. 